\title{
SATURATION PROPERTIES OF IDEALS IN GENERIC EXTENSIONS. I
}

BY

\author{
JAMES E. BAUMGARTNER ${ }^{1}$ AND ALAN D. TAYLOR ${ }^{2}$
}

\begin{abstract}
We consider saturation properties of ideals in models obtained by forcing with countable chain condition partial orderings. As sample results, we mention the following. If $M[G]$ is obtained from a model $M$ of $\mathrm{GCH}$ via any $\sigma$-finite chain condition notion of forcing (e.g. add Cohen reals or random reals) then in $M[G]$ every countably complete ideal on $\omega_{1}$ is $\omega_{3}$-saturated. If " $\sigma$-finite chain condition" is weakened to "countable chain condition," then the conclusion no longer holds, but in this case one can conclude that every $\omega_{2}$-generated countably complete ideal on $\omega_{1}$ (e.g. the nonstationary ideal) is $\omega_{3}$-saturated. Some applications to $\mathscr{P}_{\omega_{1}}\left(\omega_{2}\right)$ are included and the role played by Martin's Axiom is discussed. It is also shown that if these weak saturation requirements are combined with some cardinality constraints (e.g. $\left.2^{\aleph_{1}}>\left(2^{\aleph_{0}}\right)^{+}\right)$, then the consistency of some rather large cardinals becomes both necessary and sufficient.
\end{abstract}

0. Introduction. Suppose $I$ is a $\kappa$-complete (proper) ideal on $\kappa$ and $P$ is a notion of forcing satisfying the $\kappa$-chain condition. Then it is well known that $I$ generates a (proper) $\kappa$-complete ideal on $\kappa$ in the generic extension obtained by forcing with $P$. The results we obtain in this paper and its sequel [BT] are motivated by (but not limited to) the following type of question. For a given cardinal $\lambda$, what properties of $I$ and $P$ guarantee that this ideal generated by $I$ in the generic extension is $\lambda$-saturated?

Considerations of this type of question are not new to the literature. For example, it is shown in $\left[\mathbf{B}_{1}\right]$ that one cannot always obtain $2^{\aleph_{1}}$ almost-disjoint subsets of $\omega_{1}$ of size $\boldsymbol{\aleph}_{1}$. The proof of this result (which we will greatly generalize in what follows) actually shows that if $I=I_{\omega_{1}}$ (the ideal of countable subsets of $\left.\omega_{1}\right)$, and $\lambda=\left(2^{\aleph_{0}}\right)^{+}$ as computed in the ground model, then the answer to the question in paragraph one is "none". Hence, one obtains the consistency of $I_{\omega_{1}}$ being $\omega_{3}$-saturated while $2^{\aleph_{1}}$ is arbitrarily large by simply blowing up the continuum in any model of $\mathrm{CH}$ via any c.c.c. notion of forcing.

Another example of this type of result is the well-known theorem of Prikry [P] asserting that if $\lambda<\kappa$ and $I$ is a $\lambda$-saturated $\kappa$-complete ideal on $\kappa$, then $I$ generates a $\lambda$-saturated $\kappa$-complete ideal on $\kappa$ in any $\lambda$-c.c. generic extension. Hence, for example, if $\kappa$ is measurable and one blows up the continuum to $\kappa$ via any c.c.c. notion of forcing, then one obtains a model in which there is an $\omega_{1}$-saturated

\footnotetext{
Received by the editors October 3, 1979 and, in revised form, May 26, 1980. 1980 Mathematics Subject Classification. Primary 03E35, 03E05, 04A20.

${ }^{1}$ Research partially supported by NSF grant MCS 76-08231.

${ }^{2}$ Research partially supported by NSF grant MCS 77-04147.
} 
$2^{\omega}$-complete ideal on the real line. Prikry's result has since been generalized by Solovay [S] and Kakuda [K]. Their strengthened versions yield the preservation of (respectively) $\kappa$-saturation and $\kappa^{+}$-saturation of $\kappa$-complete ideals on $\kappa$ when forcing with $\lambda$-c.c. notions of forcing for $\lambda<\kappa$. For more results along these lines we refer the reader to [Kun, KP, W, BTW $\mathbf{W}_{1}$.

The distinction between the type of results in paragraph two above as opposed to those in paragraph three is that the former require no large cardinal assumptions, while the latter of necessity do. This distinction is also what separates the present paper from its sequel [BT], although supercompact cardinals make a brief intrusion during the discussions in $\$ 5$ of this work.

Our notation and terminology is explained in $\S 1$, while the main results of the paper begin in $\$ 2$. We show here, for example, that if $M[G]$ is obtained from a model $M$ of $\mathrm{GCH}$ via any countable chain condition notion of forcing, then in $M[G]$ every $\omega_{2}$-generated countably complete ideal on $\omega_{1}$ is $\omega_{3}$-saturated. This generalizes the result in paragraph two above, and the proof provides a rather nice application of a canonical partition relation.

In $\S 3$ we consider some applications of the results in $\$ 2$ to $\mathscr{P}_{\omega_{1}}\left(\omega_{2}\right)$. For example, we prove here that it is relatively consistent with ZFC that $2^{\kappa_{0}}$ is as large as desired and yet there is a stationary set $S \subseteq \mathscr{P}_{\omega_{1}}\left(\omega_{2}\right)$ that cannot be decomposed into $\boldsymbol{\aleph}_{4}$ pairwise disjoint stationary subsets of $S$. By way of contrast, we prove here also that $\mathscr{P}_{\omega_{1}}\left(\omega_{2}\right)$ itself can always be decomposed into $2^{\kappa_{0}}$ pairwise disjoint stationary sets.

Most of the standard c.c.c. partial orderings for enlarging the continuum (e.g. Cohen reals or random reals) turn out, in fact, to satisfy the $\sigma$-finite chain condition. This stronger chain condition assumption yields a rather striking improvement in the results of $\$ 2$. For example, we show in $\$ 4$ that if $M[G]$ is obtained from a model $M$ of $\mathrm{GCH}$ via any $\sigma$-finite c.c. notion of forcing, then in $M[G]$ every countably complete ideal on $\omega_{1}$ is $\omega_{2}$-generated, and hence $\omega_{3}$-saturated. Some independence results concerning the number of countably complete ideals on $\omega_{1}$ follow rather easily from this.

The problem of simultaneously obtaining some weak saturation requirements (e.g. the $\omega_{3}$-saturation of $I_{\omega_{1}}$ ) and some prescribed cardinality conditions (e.g. $2^{\kappa_{1}}>$ $\left.\left(2^{\aleph_{0}}\right)^{+}\right)$is discussed in $\S 5$, and this is shown to be a large cardinal problem: the consistency of a supercompact cardinal suffices to construct such a model, while the existence of such a model implies the consistency of a measurable cardinal.

Comparing the results of $\$ \S 2$ and 4 suggests that maybe the stronger saturation conclusion (i.e. the $\omega_{3}$-saturation of every countably complete ideal on $\omega_{1}$ ) could be obtained from the weaker chain condition assumption (i.e. the c.c.c. as opposed to the $\sigma$-finite c.c.). In $\$ 6$ we use a variant of a partial ordering invented by Galvin and Hajnal and show that this is not the case. In fact, $M A_{\omega_{3}}$ directly implies that there is a non- $\omega_{3}$-saturated countably complete ideal on $\omega_{1}$, while the same conclusion turns out to be independent of $M A_{\omega_{2}}$.

Finally, in $\$ 7$ we discuss some generalizations of our results and we list several open questions.

We would like to thank the referee for several suggestions that have been incorporated into the final version of this paper. 
1. Notation and terminology. Our set theoretic notation and terminology is standard. If $X$ is a set then $\mathscr{P}(X)$ is the power set of $X$ and $|X|$ is the cardinality of $X$. If $Y$ is also a set then ${ }^{X} Y$ denotes the set of all functions mapping $X$ into $Y$. If $X$ is a set and $\kappa$ is a cardinal then

$$
[X]^{\kappa}=\{Y \subseteq X:|Y|=\kappa\} \text { and }[X]^{<\kappa}=\{Y \subseteq X:|Y|<\kappa\} .
$$

If $\lambda$ is also a cardinal then $[\lambda]^{<\kappa}$ is sometimes denoted by $\mathscr{P}_{\kappa}(\lambda)$. Throughout, $\kappa, \lambda$, and $\mu$ will denote infinite cardinals and $\kappa$ will henceforth be reserved for one that is of uncountable cofinality.

A partially ordered set $P$ satisfies the $\mu$-chain condition ( $\mu$-c.c.) iff every pairwise incompatible subset of $P$ is of cardinality less than $\mu$. The $\omega_{1}$-chain condition is called the countable chain condition (c.c.c.). $P$ has the $\mu$-finite chain condition iff there is a function $f: P \rightarrow \mu$ such that for each $\alpha<\mu$, every pairwise incompatible subset of $f^{-1}(\{\alpha\})$ is finite. The $\omega$-finite chain condition is usually called the $\sigma$-finite chain condition ( $\sigma$-finite c.c.).

An ideal on $\kappa$ is said to be $\lambda$-complete if it is closed under unions of size less than $\lambda$. If $I$ is $\omega_{1}$-complete, then $I$ is also called countably complete. Throughout this paper we use the phrase "ideal on $\kappa$ " to mean one that is countably complete, proper (i.e. $\kappa \notin I)$, and contains all singleton subsets of $\kappa$. We will occasionally speak of ideals on index sets other than a cardinal (e.g. $\mathscr{P}_{\kappa}(\lambda)$ ), but the reader should have no difficulty in interpreting our notation and terminology in these contexts.

If $I$ is an ideal on $\kappa$, then $I^{+}$denotes the sets of positive I-measure; i.e. $I^{+}=\mathscr{P}(\kappa)-I$. If $A \in I^{+}$, then the restriction of $I$ to $A$ is the ideal $I \mid A=\{X \subseteq$ $\kappa: X \cap A \in I\}$. If $\left\langle X_{\alpha}: \alpha<\kappa\right\rangle$ is a sequence of subsets of $\kappa$, then the diagonal union of the sequence, denoted $\nabla\left\langle X_{\alpha}: \alpha<\kappa\right\rangle$, is defined to be

$$
\cup\left\{X_{\alpha}-(\alpha+1): \alpha<\kappa\right\} \text {. }
$$

It is well known (see [BTW $]$ ) that if $f: \kappa \rightarrow \kappa$ is a bijection, then $\nabla\left\langle X_{\alpha}: \alpha<\kappa\right\rangle$ and $\nabla\left\langle X_{f(\alpha)}: \alpha<\kappa\right\rangle$ differ only by a nonstationary subset of $\kappa$. An ideal $I$ on $\kappa$ is said to be normal if it is closed under diagonal unions; equivalently, $I$ is normal iff every regressive function on a set of positive $I$-measure is constant on a set of positive $I$-measure. $I_{\kappa}$ denotes the ideal of subsets of $\kappa$ of cardinality less than $\kappa$ and $N S_{\kappa}$ denotes the ideal of nonstationary subsets of $\kappa$. Fodor's theorem [F] asserts that if $\kappa$ is regular, then $N S_{\kappa}$ is a normal ideal on $\kappa$.

An ideal $I$ on $\kappa$ is said to be generated by the set $\mathscr{X}$ iff $I$ is the smallest ideal on $\kappa$ such that $\mathscr{X} \subseteq I$. In this case it is easy to see that $I=\left\{Z \subseteq \kappa:\left(\exists \mathscr{Y} \in[\mathscr{X}]^{\omega}\right)\right.$ $\left.\left(|Z-\cup \mathcal{Y}| \leqslant \aleph_{0}\right)\right\}$. Note that if we say "let $I$ be the ideal on $\kappa$ generated by $\mathcal{X}$," then we are asserting that $I$ exists, i.e. that $\kappa-\cup \mathcal{Y}$ is uncountable whenever $\mathcal{Y} \in[\mathcal{X}]^{\omega}$. If $I$ is generated by a set $\mathcal{X}$ of size $\lambda$ (of size less than $\lambda$ ), then we say that $I$ is $\lambda$-generated ( $<\lambda$-generated). Two sets $A$ and $B$ in $I^{+}$are said to be $I$-almost disjoint iff $A \cap B \in I$. The ideal $I$ is $\lambda$-saturated iff every pairwise $I$-almost disjoint collection $\mathscr{F} \subseteq I^{+}$is of cardinality less than $\lambda$. Note that $I$ is $\lambda$-saturated iff the Boolean algebra $\mathscr{P}(\kappa) / I$ satisfies the $\lambda$-chain condition. For more background on the theory of ideals, see $\left[\mathbf{J P}, \mathbf{B T W}_{2}, \mathbf{T}_{1}\right]$ or $\left[\mathbf{T}_{2}\right]$. 
We assume that the reader is familiar with the theory of forcing, and our notation in these matters is reasonably standard. Notice, however, that we write $p \leqslant q$ to mean that $p$ contains more information than $q$. Hence, a set $D \subseteq P$ is dense iff for every $p \in P$ there exists $d \in D$ such that $d \leqslant p$. We also generally discuss forcing as if it is taking place over the universe of set theory $V$. The reader uncomfortable with this approach can easily recast these results in terms of a countable transitive model $M$ of a sufficiently large fragment of $\mathrm{ZFC}$.

2. Positive preservation results for c.c.c. extensions. Our first few results here establish some connections between the number of generators of an ideal and its degree of saturation. The following, for example, shows that the existence of a non- $\lambda$-saturated ideal on $\kappa$ requires the existence of an ideal on $\kappa$ that is not $<\lambda$-generated.

THEOREM 2.1. (a) If every ideal on $\kappa$ is $<\lambda$-generated, then every ideal on $\kappa$ is $\lambda$-saturated.

(b) If $\kappa$ is regular and every normal ideal on $\kappa$ is $<\lambda$-generated, then every normal ideal on $\kappa$ is $\lambda$-saturated.

Proof. (a) Suppose $I$ is not $\lambda$-saturated. If $\lambda \leqslant \operatorname{cf}(\kappa)$ then $I_{\kappa}$ shows that not every ideal on $\kappa$ is $\left\langle\lambda\right.$-generated. If $\lambda>\operatorname{cf}(\kappa)$ then let $\left\langle A_{\alpha}: \alpha<\lambda\right\rangle$ be a sequence of pairwise $I$-almost disjoint elements of $I^{+}$. Let $J$ be the ideal on $\kappa$ generated by the set $\left\{A_{\alpha}: \alpha<\lambda\right\}$. Then $J$ is not generated by any set of cardinality less than $\lambda$, since if $\alpha \in \lambda$ and $X$ is a countable subset of $\lambda-\{\alpha\}$, then $A_{\alpha} \nsubseteq \cup\left\{A_{\beta}: \beta \in X\right\}$.

The proof of (b) is similar, except that $I_{\kappa}$ is replaced by $N S_{\kappa}$ and $J$ is replaced by the smallest normal ideal containing $\left\{A_{\alpha}: \alpha<\lambda\right\}$.

We have been unable to prove the converse of Theorem 2.1(a).

In a preliminary version of this paper we asked if Theorem 2.1(b) could be strengthened from its present form (in which both the hypothesis and the conclusion speak about all normal ideals on $\kappa$ ) to one that considers only a single normal ideal $I$ on $\kappa$. That is, if $\kappa$ is regular and $I$ is a normal ideal on $\kappa$ that is $<\lambda$-generated, must $I$ be $\lambda$-saturated? The answer to this was provided independently by T. Jech $\left[\mathbf{J}_{\mathbf{2}}\right]$ and A. Kanamori [Ka]. Each provided a model in which $N S_{\omega_{1}}$ is $\omega_{2}$-generated but not $\omega_{3}$-saturated. Nevertheless, the following result shows that in certain models of set theory it does turn out that every $\lambda$-generated ideal $I$ on $\kappa$ (whether $I$ is normal or not) is $\lambda^{+}$-saturated.

THEOREM 2.2. Assume $\mathrm{GCH}$, and let $P$ be a partial ordering with the countable chain condition. Assume $\kappa$ and $\lambda$ are cardinals such that $\omega<\kappa<\operatorname{cf}(\lambda)$. Then $\Vdash_{P}$ "If I is an ideal on $\kappa$ which is $\lambda$-generated, then $\dot{I}$ is $\lambda^{+}$-saturated."

Proof. Assuming GCH we have the following partition relation (see $\left[\mathbf{B}_{\mathbf{2}}\right.$, Theorem 1] for the case where $\lambda$ is regular and [EHMR, Lemma 15.2] for the general case): if $f:\left[\lambda^{+}\right]^{2} \rightarrow \lambda$ then there is $Z \subseteq \lambda^{+}$such that $|Z|=\kappa^{+}$and if $\alpha, \beta, \gamma \in Z$ with $\alpha<\beta, \gamma$, then $f(\{\alpha, \beta\})=f(\{\alpha, \gamma\})$.

Now suppose $\mathbb{}_{P} "\left\langle\dot{B}_{\alpha}: \alpha<\lambda\right\rangle$ generates $\dot{I}$ and $\left\langle\dot{A}_{\alpha}: \alpha<\lambda^{+}\right\rangle$is an $\dot{I}$-almost disjoint sequence of elements of $\dot{I}^{+}$". If $\alpha, \beta<\lambda^{+}$then, since $P$ has the c.c.c., there 
is a countable set $X=X(\alpha, \beta) \subseteq \lambda$ such that $\mathbb{}_{P}$ " $\dot{A}_{\alpha} \cap \dot{A}_{\beta} \subseteq \cup\left\{\dot{B}_{\gamma}: \gamma \in X\right\}$." There are at most $\lambda^{\omega}=\lambda$ such sets $X$, so by the partition theorem described above, there is $Z \subseteq \lambda^{+}$such that $|Z|=\kappa^{+}$and if $\alpha, \beta, \gamma \in Z$ with $\alpha<\beta, \gamma$, then $X(\alpha, \beta)$ $=X(\alpha, \gamma)$. For $\alpha \in Z$ let $X_{\alpha}$ denote the constant value of $X(\alpha, \beta)$ for $\beta \in Z$ with $\alpha<\beta$. For $\alpha \in Z$ let $\dot{C}_{\alpha}$ be a term denoting $\dot{A}_{\alpha}-\cup\left\{\dot{B}_{\beta}: \beta \in X_{\alpha}\right\}$. Now, for all $\alpha \in Z, \mathbb{}_{P} \dot{C}_{\alpha} \in \dot{I}^{+}$, but at the same time, for all $\alpha, \beta \in Z$ with $\alpha \neq \beta, \mathbb{}_{P} \dot{C}_{\alpha} \cap \dot{C}_{\beta}$ $=0$. Since $|Z|=\kappa^{+}$and cardinals are preserved under forcing with $P$, this asserts the existence of $\kappa^{+}$pairwise disjoint nonempty subsets of $\kappa$, which is impossible.

If $\kappa=\omega_{1}$ and $\lambda=\omega_{2}$, Theorem 2.2 can be immediately applied to the ideals $I_{\omega_{1}}$ and $N S_{\omega_{1}}$.

COROLlaRY 2.3. If $\mathrm{GCH}$ holds and $P$ has the countable chain condition, then

(a) $\Vdash_{P}$ " $I_{\omega_{1}}$ is $\omega_{3}$-saturated," and

(b) $\Vdash_{P}$ " $N S_{\omega_{1}}$ is $\omega_{3}$-saturated."

Proof. Part (a) is clear, and is an old result of Baumgartner [ $\left.\mathbf{B}_{1}\right]$. To prove (b), simply use the well-known result that if $P$ has the c.c.c., then $\mathbb{F}_{P}$ "Every element of $N S_{\omega_{1}}$ is contained in an element of $N S_{\omega_{1}}^{V}$ " " Since $\left|N S_{\omega_{1}}^{V}\right|=\omega_{2}$, we have $\Vdash_{P}$ " $N S_{\omega_{1}}$ is generated by $\omega_{2}$ sets."

There are also non-GCH versions of the partition relation used in the proof of Theorem 2.2 above. For example, it is shown in $\left[\mathbf{B}_{2}\right.$, Theorem 1] that if $f$ : $\left[\left(2^{\aleph_{1}}\right)^{+}\right]^{2} \rightarrow 2^{\aleph_{1}}$ then there is a set $Z \subseteq\left(2^{\aleph_{1}}\right)^{+}$such that $|Z|=\aleph_{2}$ and if $\alpha, \beta, \gamma \in Z$ with $\alpha<\beta, \gamma$, then $f(\{\alpha, \gamma\})=f(\{\beta, \gamma\})$. Using this, one obtains the following preservation result from Theorem 2.2.

Corollary 2.4. Suppose $M$ is a countable transitive model of ZFC and $M$ F " $\left(2^{\aleph_{1}}\right)^{+}=\kappa$ and $I$ is a countably complete ideal on $\omega_{1}$." Then $I$ is (obviously) $\kappa$-saturated and, moreover, I generates a $\kappa$-saturated countably complete ideal on $\omega_{1}$ in any c.c.c. generic extension of $M$.

Loosely speaking, Corollary 2.4 asserts that the $\left(2^{\aleph_{1}}\right)^{+}$-saturation of $I$ is preserved in any c.c.c. generic extension. Of course, for this to be nontrivial one must note that " $\left(2^{\aleph_{1}}\right)^{+}$" is being computed in the ground model.

3. Applications to $\mathscr{P}_{\omega_{1}}(\kappa)$. In [J], Jech generalizes the notions of closed unbounded set and stationary set to $\mathscr{P}_{\kappa}(\lambda)=[\lambda]^{<\kappa}$ as follows. A set $C \subseteq[\lambda]^{<\kappa}$ is closed if it is closed under unions of increasing sequences of fewer than $\kappa$ of its elements. $C$ is unbounded if for every $X \in[\lambda]^{<\kappa}$ there exists $Y \in C$ such that $X \subseteq Y$. Finally, a set $S \subseteq[\lambda]^{<\kappa}$ is stationary if $S \cap C \neq 0$ for every closed unbounded set $C \subseteq[\lambda]^{<\kappa}$.

It is easy to see that if $f:[\kappa]^{<\omega} \rightarrow \kappa$ and $C_{f}=\left\{X \in[\kappa]^{\omega}: X\right.$ is closed under $\left.f\right\}$, then $C_{f}$ is a closed unbounded set in $[\kappa]^{\omega}$. The following partial converse of this observation is essentially due to Kueker [Ku]: if $C \subseteq[\kappa]^{\omega}$ is closed unbounded, then there exists a function $f:[\kappa]^{<\omega} \rightarrow \kappa$ such that $C_{f} \subseteq C$. For the sake of completeness, we sketch the proof. For $1 \leqslant n<\omega$ let $h_{n}:[\kappa]^{n} \rightarrow C$ be defined by induction on $n$ as follows. If $n=1$, then let $h_{n}(\{x\})$ be any set $X \in C$ such that $x \in X$. Now given $t \in[\kappa]^{n+1}$ let $h_{n+1}(t)$ be any set $Y \in C$ so that $h_{|s|}(s) \subseteq Y$ for every $s \subseteq t$ with $1 \leqslant|s| \leqslant n$. Now for $1 \leqslant n<\omega$ define $g_{n}:[\kappa]^{<\omega} \rightarrow \kappa$ as follows: if $|s|=k$ then let 
$g_{n}(s)$ be the $n$th point of $h_{k}(s)$ where $h_{k}(s)$ has been enumerated in order-type $\omega$. Define $g_{0}(s)=\sup (s)+1$, and let $r: \omega \times \omega \rightarrow \omega$ be any bijection. Finally, the desired function $f:[\kappa]^{<\omega} \rightarrow \kappa$ is obtained as follows. If $s=\left\{x_{0}, \ldots, x_{n+1}\right\}$ and $r^{-1}(n)=(p, q)$, then let $f(s)=g_{p}\left(\left\{x_{0}, \ldots, x_{q-1}\right\}\right)$. It is not difficult to check that $f$ works.

To put the results of this section in perspective, we mention the following known decomposition results for stationary subsets of $[\lambda]^{<\kappa}$. Jech showed in $[\mathbf{J}]$ that if $\kappa$ is a successor cardinal and $\lambda \geqslant \kappa$ is regular, then every stationary set $S \subseteq[\lambda]^{<\kappa}$ can be decomposed into $\lambda$ pairwise disjoint stationary subsets. Thus, for example, if $S$ is stationary in $\left[\omega_{2}\right]^{\omega}$, and $I$ denotes the ideal of nonstationary subsets of $\left[\omega_{2}\right]^{\omega}$, then $I \mid S$ is not $\omega_{2}$-saturated. Diprisco [Di] showed that if $\kappa$ is a regular limit cardinal and $\lambda \geqslant \kappa$ is regular, then $[\lambda]^{<\kappa}$ itself can be decomposed into $\lambda$ pairwise disjoint stationary sets. Whether or not such a decomposition can be obtained for every stationary subset of $[\lambda]^{<\kappa}$ in this case is still open, although Baumgartner has shown (unpublished) that a negative answer implies the existence of $0^{\#}$.

The following theorem imposes some limitations on any improvements of the above results.

Theorem 3.1. Assume $\mathrm{GCH}$, and let $\lambda=\kappa^{\omega}$. Let $P$ be any c.c.c. partial ordering and let $\dot{I}$ be a term denoting the nonstationary ideal on $[\kappa]^{\omega}$ in the extension. Then

${ }{ }_{P}$ "There is a stationary set $S \subseteq[\kappa]^{\omega}$ such that $|S|=\lambda$ and $\dot{I} \mid S$ is $\lambda^{++}$-saturated."

Proof. Let $S=\left([\kappa]^{\omega}\right)^{V}$. Then clearly $|S|=\lambda$. We must check that $\Vdash_{P}$ " $S$ is stationary." Suppose $\mathbb{}_{P}$ " $\dot{C} \subseteq[\kappa]^{\omega}$ is closed unbounded." We may assume that $\dot{f}$ is such that $\mathbb{F}_{P}$ " $\dot{f}:[\kappa]^{<\omega} \rightarrow \kappa$ and $\dot{C}=\left\{X \in[\kappa]^{\omega}: X\right.$ is closed under $\left.\dot{f}\right\}$." Since $P$ has the c.c.c. there is some $g \in V$ such that $g:[\kappa]^{<\omega} \rightarrow[\kappa]^{\omega}$ and for all $s$, $\mathbb{F}_{P} \dot{f}(s) \in g(s)$.

Let $C=\left\{X \in[\kappa]^{\omega}: \forall s \in[X]^{<\omega} g(s) \subseteq X\right\}$. Then $C$ is closed unbounded in $V$ and $\Vdash_{P} C \subseteq \dot{C}$. Thus $\mathbb{}_{P} \dot{C} \cap S \neq 0$, as desired. But also, if $\bar{C}$ is such that $\Vdash_{P} \bar{C}$ is the closure of $C$, then $\Vdash_{P} \bar{C} \subseteq \dot{C}$ and $\bar{C} \cap S=C \cap S$. Hence

\section{$\Vdash_{p}$ "the nonstationary sets in $V$ generate $i \mid S$."}

It follows that $\mathbb{}_{P}$ " $i \mid S$ is $\lambda^{+}$-generated" and, by Theorem $2.2, \quad \mathbb{t}_{P}$ " $\dot{I} \mid S$ is $\lambda^{++}$-saturated."

Remark. For $\kappa=\omega_{n}, n<\omega$, it is possible to prove in ZFC that there is a stationary subset of $[\kappa]^{\omega}$ of power $\kappa$. For $\kappa=\omega_{2}$ we proceed as follows. For each $\alpha<\omega_{2}$ let $f_{\alpha}: \alpha \rightarrow \omega_{1}$ be 1 to 1 . If $\alpha<\omega_{2}$ and $\xi<\omega_{1}$ let $X_{\alpha \xi}=\left\{\beta<\alpha: f_{\alpha}(\beta)<\xi\right\}$. Let $S=\left\{X_{\alpha \xi}: \alpha<\omega_{2}, \xi<\omega_{1}\right\}$. It is not difficult to see that $S$ is stationary. A similar approach works for $\kappa=\omega_{n}, n>2$. We do not know whether it is provable that for $\kappa \geqslant \omega_{\omega}$ there is a stationary subset of $[\kappa]^{\omega}$ of power $\kappa$.

In view of Theorem 3.1, it is natural to ask whether under the same hypotheses we have $\Vdash_{P}$ " $\dot{I}$ is $\lambda^{++}$-saturated." The answer, rather surprisingly, is negative. See Theorem 3.4 below.

THEOREM 3.2. (a) If $C$ is a closed unbounded subset of $\left[\omega_{2}\right]^{\omega}$, then there is a countable set $A \subseteq \omega_{2}$ such that $\left|[A]^{\omega} \cap C\right|=2^{\aleph_{0}}$.

(b) If $Q$ is a structure of cardinality $\aleph_{2}$ with countably many relations and operations, then there is a countable $\mathscr{B}<\mathbb{Q}$ such that $\mathscr{B}$ has $2^{\aleph_{0}}$ elementary substructures. 
Proof. Notice first that (b) simply restates (a). In proving (a), we can assume without loss of generality that for some $f:\left[\omega_{2}\right]^{<\omega} \rightarrow \omega$ we have $C=\left\{X \in\left[\omega_{2}\right]^{\omega}: X\right.$ is closed under $f\}$. For $A \subseteq \omega_{2}$ let $\operatorname{cl}(A)$ denote the closure of $A$ under $f$. Now $Z=\left\{\alpha<\omega_{2}: \alpha\right.$ is closed under $\left.f\right\}$ is closed unbounded in $\omega_{2}$. If $\alpha \in Z$ and $\operatorname{cf}(\alpha)=\omega$, let $\left\langle\xi_{n}^{\alpha}: n \in \omega\right\rangle$ be an increasing sequence cofinal in $\alpha$ and let $A_{\alpha}=$ $\operatorname{cl}\left(\left\{\xi_{n}^{\alpha}: n \in \omega\right\}\right)$.

If $n \in \omega$ and $s \in{ }^{n} 2$ then let $s \frown i$ denote $s \cup\{(n, i)\}$. Now for each $s \in \cup_{n \in \omega}{ }^{n} 2$, we will define an ordinal $\xi_{s}$ and a stationary set $Z_{s} \subseteq\{\alpha \in Z: \operatorname{cf}(\alpha)=\omega\}$ such that (1)-(3) below hold.

(1) $\forall \alpha \in Z_{s} \exists n \in \omega\left(\xi_{s}=\xi_{n}^{\alpha}\right)$.

(2) $\forall s \forall \alpha \in Z_{s^{\wedge} 0} \forall \beta \in Z_{s^{\wedge} 1}\left(\xi_{s^{\wedge} 0} \notin A_{\beta}\right.$ and $\left.\xi_{s^{\wedge} 1} \notin A_{\alpha}\right)$.

(3) $\forall s\left(Z_{s^{\sim 0}} \cup Z_{s^{\wedge} 1} \subseteq Z_{s}\right)$.

This will complete the proof, for if $f$ and $g$ are distinct members of ${ }^{\omega} 2$ then $\operatorname{cl}\left(\left\{\xi_{f \mid n}: n \in \omega\right\}\right)$ and $\operatorname{cl}\left(\left\{\xi_{g \mid n}: n \in \omega\right\}\right)$ are clearly distinct (in fact incomparable) subsets of $A=\operatorname{cl}\left(\left\{\xi_{s}: s \in \cup_{n \in \omega}{ }^{n} 2\right\}\right)$.

The construction of $\xi_{s}$ and $Z_{s}$ takes place by induction on $|s|$. Given $\xi_{s}$ and $Z_{s}$ we proceed as follows.

First we claim that $K=\left\{\xi<\omega_{2}:\left\{\alpha \in Z_{s}: \exists n\left(\xi=\xi_{n}^{\alpha}\right)\right\}\right.$ is stationary $\}$ has cardinality $\boldsymbol{\aleph}_{2}$. If not then there is $\xi$ such that $\sup (K)<\xi<\omega_{2}$. For $\alpha \in Z_{s}, \alpha>\xi$, let $h(\alpha)$ be the least element of $\left\{\xi_{n}^{\alpha}: n \in \omega\right\}$ which is greater than or equal to $\xi$. Then $h$ is regressive so $h$ is constant on a stationary set, contrary to the choice of $\xi$. Thus $|K|=\boldsymbol{\aleph}_{2}$. Let $K^{\prime}$ consist of the first $\omega_{1}$ elements of $K$. Now for every $\alpha \in Z_{s}$ there is some $\xi \in K^{\prime}$ such that $\xi \notin A_{\alpha}$. Thus for every $\xi \in K$ there is $\eta=g(\xi) \in K^{\prime}$ and stationary $S_{\xi} \subseteq\left\{\alpha \in Z_{s}: \xi \in\left\{\xi_{n}^{\alpha}: n \in \omega\right\}\right\}$ such that for every $\alpha \in S_{\xi}$ we have $\eta \in A_{\alpha}$. Hence there is $K_{1} \subseteq K,\left|K_{1}\right|=\aleph_{2}$ such that $g$ is constant on $K_{1}$, say $g\left(K_{1}\right)=\left\{\eta_{0}\right\}$.

But now by a similar argument we can find stationary $T \subseteq\left\{\alpha \in Z_{s}: \eta_{0} \in\left\{\xi_{n}^{\alpha}\right.\right.$ : $n \in \omega\}\}$ and $\eta_{1} \in K_{1}$ so that for every $\alpha \in T, \eta_{1} \notin A_{\alpha}$. Now let $\xi_{s\urcorner 0}=\eta_{0}$, $\xi_{s^{\wedge} 1}=\eta_{1}, Z_{s^{\urcorner}}=T$ and $Z_{s^{\wedge} 1}=S_{\eta_{1}}$.

COROLlary 3.3. Suppose $\kappa \geqslant \omega_{2}$. Then every closed unbounded subset of $[\kappa]^{\omega}$ has cardinality $\kappa^{\omega}$.

Proof. First suppose that $\kappa \leqslant 2^{\omega}$. Then $\kappa^{\omega}=2^{\omega}$. If $C \subseteq[\kappa]^{\omega}$ is closed unbounded then it is easy to see that there is $X \subseteq \kappa,|X|=\boldsymbol{\aleph}_{2}$, such that $C \cap[X]^{\omega}$ is closed unbounded in $[X]^{\omega}$ (in the obvious sense). Now apply Theorem 3.2.

If $2^{\omega}<\kappa$, then for each $X \in[\kappa]^{\omega}$ there is $f(X) \in C$ with $X \subseteq f(X)$. The map $f$ has the property that $\left|f^{-1}(Y)\right| \leqslant 2^{\aleph_{0}}$ for each $Y \in C$, so there must be $\kappa^{\omega}$ distinct elements of $C$. Note that only the unboundedness of $C$ was used in this case.

THEOREM 3.4. If $\omega_{2} \leqslant \kappa \leqslant 2^{\omega}$, then there exist $2^{\aleph_{0}}$ pairwise disjoint stationary subsets of $[\kappa]^{\omega}$.

Proof. We assume $\kappa=\omega_{2}$ and leave the rest to the reader. Let $\left\langle\left(A_{\alpha}, f_{\alpha}\right): \alpha<2^{\omega}\right\rangle$ enumerate all pairs $(A, f)$ such that $A \in\left[\omega_{2}\right]^{\omega}, f:[A]^{<\omega} \rightarrow A$ and $A$ has $2^{\kappa_{0}}$ subsets closed under $f$. It is easy now to construct disjoint $S_{\beta}$ for $\beta<2^{\omega}$ such that for every 
$\alpha$ and $\beta$ there is $B \in S_{\beta}, B \subseteq A_{\alpha}$ and $B$ is closed under $f$. Now, by Theorem 3.2, each $S_{\beta}$ is stationary.

In $\left[\mathbf{M e}_{\mathbf{1}}\right]$, Menas conjectures that if $\kappa$ is regular and $\kappa<\lambda$ then every stationary subset of $[\lambda]^{<\kappa}$ can be split into $\lambda^{k}$ disjoint stationary subsets. The following consequence of the results in this section disproves this conjecture, but it partially affirms it at the same time.

COROLlary 3.5. It is relatively consistent with ZFC that $2^{\omega}$ is large and the nonstationary ideal I on $\left[\omega_{2}\right]^{\omega}$ is not $2^{\omega}$-saturated, whereas there is a stationary set $S \subseteq\left[\omega_{2}\right]^{\omega}$ such that $I \mid S$ is $\omega_{4}$-saturated.

Proof. If one begins with a model of GCH and then enlarges the continuum by forcing with a c.c.c. partial ordering, then the result follows immediately from Theorems 3.1 and 3.4 .

4. Positive preservation results for $\sigma$-finite c.c. extensions. It turns out that most of the familiar c.c.c. partial orderings can be used to produce models in which every ideal on $\omega_{1}$ is $\omega_{3}$-saturated. The reason for this is that they often satisfy the $\sigma$-finite chain condition. Note, for example, that this is true of the orderings for enlarging the continuum with either Cohen reals or random reals.

THEOREM 4.1. Suppose $2^{\kappa}=\kappa^{+}$and $P$ has the $\sigma$-finite chain condition. Then $\mathbb{}_{P}$ "Every ideal on $\kappa$ is $\kappa^{+}$-generated and hence $\kappa^{++}$-saturated."

Proof. Suppose $\lambda>\kappa^{+}$and $\Vdash_{P}$ " $\left\langle\dot{A}_{\alpha}: \alpha<\lambda\right\rangle$ is a sequence of generators for $\dot{I}$." Form a structure $Q=(\lambda \cup P, \leqslant, f, R, \xi)_{\xi<\kappa}$ where $\leqslant$ is the ordering on $P$, $f: P \rightarrow \omega$ witnesses the $\sigma$-finite chain condition for $P$, and $R(\alpha, \xi, p)$ iff $p \Vdash \xi \in \dot{A}_{\alpha}$. Endow $\mathscr{Q}$ with Skolem functions. Let $\mathscr{B}$ be a relatively $\omega$-saturated elementary substructure of $Q$; i.e., if $F \subseteq \mathscr{B}$ is finite, then every type over $(\mathscr{B}, \alpha)_{\alpha \in F}$ which is realized in $\mathscr{Q}$ is realized in $\mathscr{B}$. It is well known that there is such $\mathscr{B}$ with $|\mathscr{B}| \leqslant 2^{\kappa}=\kappa^{+}$. Let $X=\lambda \cap \mathscr{B}$. We claim that $\Vdash_{P}$ " $\forall \alpha \in \lambda \exists Y \in[X]^{\omega} \cap V$ such that $\dot{A}_{\alpha} \subseteq$ $\cup\left\{\dot{A}_{\beta}: \beta \in Y\right\}$." This immediately yields a set of generators for $\dot{I}$ of cardinality $\leqslant \kappa^{+}$.

Let $\alpha \in \lambda$. If $\alpha \in X$ we are done, so suppose otherwise. Let $\left\langle\alpha_{n}: n \in \omega\right\rangle$ be a sequence of elements of $X$ such that for each $n, \alpha_{n}$ realizes the same type over $\left(\Re, \alpha_{m}\right)_{m<n}$ that $\alpha$ realizes. We assert that $\Vdash_{P} \dot{A}_{\alpha} \subseteq \cup\left\{\dot{A}_{\alpha_{n}}: n \in \omega\right\}$. Suppose $p \Vdash \xi$ $\in \dot{A}_{\alpha}$. We must find $q \leqslant p$ and $n \in \omega$ so that $q \Vdash \xi \in \dot{A}_{\alpha_{n}}$.

Let $f(p)=i$. Then the statement " $\exists p(f(p)=i$ and $R(\alpha, \xi, p))$ " is true of $\alpha$ and hence must be true of $\alpha_{0}$. So there is a $p_{0} \in \Re$, definable from $\alpha_{0}$, such that $p_{0} \mathbb{H}^{\text {" }} \xi \in \dot{A}_{\alpha_{0}}$ and $f\left(p_{0}\right)=i$." If $p$ and $p_{0}$ are compatible, we are done. If not, consider the statement " $\exists p$ ( $p$ is incompatible with $p_{0}$ and $f(p)=i$ and $R(\alpha, \xi, p)$ )." This is true of $\alpha$, hence must be true of $\alpha_{1}$, so there is $p_{1} \in \Re$, definable from $\alpha_{0}$ and $\alpha_{1}$, such that $p_{1}$ is incompatible with $p_{0}, f\left(p_{1}\right)=i$ and $p_{1} \Vdash \xi \in \dot{A}_{\alpha_{1}}$. If $p_{1}$ and $p$ are compatible we are done. If not, we continue in the obvious fashion. This process cannot go on ad infinitem or $f^{-1}(i)$ would have an infinite antichain, contradicting the $\sigma$-finite chain condition. 
The fact that $\mathbb{}_{P}$ "Every ideal on $\kappa$ is $\kappa^{++}$-saturated" now follows by Theorem 2.1(a).

It is clear that the proof of Theorem 4.1 will work even for cardinal preserving $P$ with the $\omega_{1}$-finite chain condition. On the other hand, the partial ordering to be used in $\S 6$ shows that Theorem 4.1 cannot be extended even to c.c.c. orderings with the $\omega_{2}$-finite chain condition.

If one begins with a model of $\mathrm{GCH}$ and forces $M A_{\omega_{2}}$ via the usual ordering, then an argument similar to the proof of Theorem 4.1 yields the following.

THEOREM 4.2. It is relatively consistent with $M A_{\omega_{2}}$ and $2^{\aleph_{0}}$ as large as desired that all ideals on $\omega_{1}$ are $\omega_{3}$-saturated.

In fact, the argument indicated above yields a model in which all ideals on $\omega_{1}$ are $\omega_{2}$-saturated, $2^{\aleph_{0}}$ is as large as desired and full $M A$ holds when restricted to orderings of cardinality at most $\aleph_{2}$. We will show in $\$ 6$ that it is also relatively consistent with $M A_{\omega_{2}}$ that there is a non- $\omega_{3}$-saturated ideal on $\omega_{1}$ and, in fact, that the existence of such an ideal is a direct consequence of $M A_{\omega_{3}}$.

Another application of Theorem 4.1 is to the possible number of ideals on $\omega_{1}$. If every ideal on $\omega_{1}$ is $\omega_{2}$-generated, then there can only be $2^{\kappa_{2}}$ distinct ideals on $\omega_{1}$. On the other hand, $2^{\aleph_{2}}$ is the minimum such number, for if $F$ is a family of $\boldsymbol{\aleph}_{2}$ pairwise almost disjoint subsets of $\omega_{1}$ (and such an $F$ always exists), then distinct subsets of $F$ generate distinct ideals. If $\mathrm{CH}$ holds, then $F$ can be chosen with cardinality $2^{\aleph_{1}}$ so there are $2^{2^{N_{1}}}$ ideals on $\omega_{1}$, the obvious maximum number. But if the continuum is blown up with Cohen reals, then Theorem 4.1 applies and we obtain the following.

COROLlaRY 4.3. It is relatively consistent with ZFC that the continuum is large, $2^{\aleph_{2}}<2^{2^{\kappa_{1}}}$, and there are only $2^{\aleph_{2}}$ ideals on $\omega_{1}$. (Of course we can even arrange $2^{\aleph_{2}}=2^{\aleph_{0}}$.)

5. Applications to saturated ideals and the GCH. The results of the previous section show that if one starts with a model of GCH and blows up the continuum via Cohen reals or random reals, then one obtains a model in which every ideal on $\omega_{1}$ is $\omega_{3}$-saturated. Now it is well known that in the extension one has either $2^{\kappa_{0}}=2^{\kappa_{1}}$ or $\operatorname{cf}\left(2^{\aleph_{0}}\right)=\aleph_{1}$ and $2^{\aleph_{1}}=\left(2^{\aleph_{0}}\right)^{+}$. The question arises as to whether these cardinality constraints are merely a technical consequence of the particular constructions we are using, or whether these weak saturation properties really do influence cardinal exponentiation.

This question has been considered before, and the partial results obtained suggest it is really the latter that is taking place. For example, Baumgartner $\left[\mathbf{B}_{\mathbf{1}}\right]$ showed that

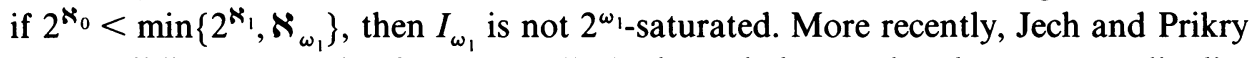
[JP], building on work of Ketonen [Ke], showed that under the same cardinality assumptions as Baumgartner's result, one can also conclude that no ideal on $\omega_{1}$ is $\lambda$-saturated for any $\lambda<2^{\aleph_{1}}$. But now, what if $2^{\aleph_{0}}>\boldsymbol{\aleph}_{\omega_{1}}$ ? Do these weak saturation properties force $2^{\aleph_{0}}$ to be the smallest permissible cardinal greater than or equal to $2^{\aleph_{1}}$ ? Our goal in this section is to show that an affirmative answer to this question lies consistency-wise between a measurable cardinal and a supercompact cardinal. 
Our starting point is the following notion, the inspiration for which is the well-known "covering lemma" of Jensen [Je]. If $M$ is an inner model of set theory, then $V$ is said to have the covering property with respect to $M$ iff $M$ is a definable class (in $V$ ) and whenever $X$ is an uncountable set of ordinals (in $V$ ), there is a set $Y \in M$ such that $X \subseteq Y$ and $|X|=|Y|$. Jensen's covering lemma [Je] asserts that $V$ has the covering property with respect to $L$ iff $0^{\#}$ does not exist. The relevance of covering properties to our considerations is shown by the following.

THEOREM 5.1. Suppose $V$ has the covering property with respect to a model $M$ of

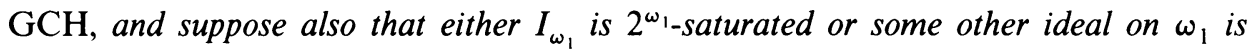
$\mu$-saturated for some $\mu<2^{\omega_{1}}$. Then either $2^{\aleph_{0}}=2^{\aleph_{1}}$ or $\operatorname{cf}\left(2^{\aleph_{0}}\right)=\omega_{1}$ and $2^{\aleph_{1}}=\left(2^{\aleph_{0}}\right)^{+}$.

Proof. Suppose that $2^{\aleph_{1}} \geqslant\left(2^{\aleph_{0}}\right)^{+}$and that if $\operatorname{cf}\left(2^{\aleph_{0}}\right)=\omega_{1}$ then $2^{\aleph_{1}} \geqslant\left(2^{\aleph_{0}}\right)^{++}$.

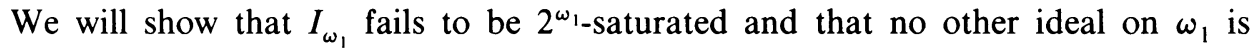
$\mu$-saturated for any $\mu<2^{\omega_{1}}$. Now, a quick glance at the proof of Theorem 6.1.1 of [JP, p. 64] shows that all we need establish is the conclusion of Theorem 5.1.1 of [JP, p. 54]. This asserts that for each regular cardinal $\mu \leqslant 2^{\omega_{1}}$ there is a "branching family" $\mathscr{F}$ of size $\mu$ consisting of functions from $\omega_{1}$ to $\omega_{1}$ (i.e. if $f, g \in \mathscr{F}$ and $f \neq g$ then for some $\gamma<\omega_{1}$ we have $f(\alpha)=g(\alpha)$ for all $\alpha<\gamma$ and $f(\alpha) \neq g(\alpha)$ for all $\alpha \geqslant \gamma)$.

To produce the desired branching family, we begin as in the Jech-Prikry proof of their Theorem 5.1. For notational simplicity, let $\kappa=2^{\omega}$ and $\lambda=2^{\omega_{1}}$. Fix a bijection $g:\left[\omega_{1}\right]^{\omega} \rightarrow \kappa$ and for each $X \subseteq \omega_{1}$ define $f_{X}: \omega_{1} \rightarrow \kappa$ by

$$
f_{X}(\alpha)=g(X \cap \alpha) \text {. }
$$

Notice that if $X \neq Y$ and $\gamma=\inf (X \triangle Y)$ then $f_{X}(\alpha)=f_{Y}(\alpha)$ for all $\alpha<\gamma$ and $f_{X}(\alpha) \neq f_{Y}(\alpha)$ for all $\alpha \geqslant \gamma$. Since $V$ has the covering property with respect to $M$ we can choose a set $X^{\prime} \in M$ for each $X \subseteq \omega_{1}$ such that $\left|X^{\prime}\right|=\omega_{1}$ and $\operatorname{range}\left(f_{X}\right) \subseteq X^{\prime}$ $\subseteq \kappa$.

Now, suppose first that $\operatorname{cf}(\kappa)>\omega_{1}$ and $\lambda>\kappa$. Then $(\operatorname{cf}(\kappa))^{M} \geqslant \omega_{2}$ and so, since $M \vDash G C H$ we have $\left|[\kappa]^{\omega_{1}} \cap M\right|=\kappa$. But now if $\mu$ is any regular cardinal such that $\kappa<\mu \leqslant \lambda$ then there must exist a set $Y \in M$ such that $|\Re| \geqslant \mu$ where $\mathscr{B}=\{X \subseteq$ $\left.\omega_{1}: X^{\prime}=Y\right\}$. It now follows easily that $\left\{f_{X}: X \in \mathscr{B}\right\}$ yields the desired branching family.

Finally, suppose $\operatorname{cf}(\kappa)=\omega_{1}$ and $\kappa^{+}<\lambda$. Then $\left|[\kappa]^{\omega_{1}} \cap M\right| \leqslant \kappa^{+}$and so we can proceed as before to obtain the desired branching family.

It should be noted that no strength is lost in the statement of Theorem 5.1 if we delete the reference to ideals on $\omega_{1}$ other than $I_{\omega_{1}}$. The point is that if some ideal on

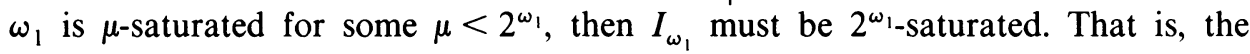
enumerating functions for sets in an almost disjoint family in $\left[\omega_{1}\right]^{\omega_{1}}$ constitute a set of eventually different functions, and well-known arguments (see [JP]) show that if one has $2^{\kappa_{1}}$ eventually different functions mapping $\omega_{1}$ to $\omega_{1}$, then no ideal on $\omega_{1}$ is $\mu$-saturated for any $\mu<2^{\aleph_{1}}$.

Theorem 5.1 shows that if we have some weak saturation and $2^{N_{1}}$ is not the smallest available cardinal $\geqslant 2^{\kappa_{0}}$, then $V$ does not have the covering property with respect to any model $M$ of $\mathrm{GCH}$. Hence, by Jensen's covering lemma, $0^{\#}$ exists. 
Moreover, recent work of Dodd and Jensen [DJ] shows that if $V$ fails to have the covering property with respect to the "core-model" $K$, then there is a measurable cardinal in an inner model. Since the core model $K$ is always a model of $\mathrm{GCH}$, we obtain the following.

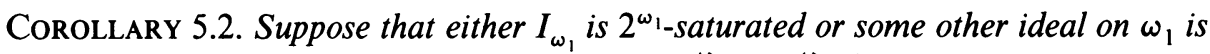
$\lambda$-saturated for some $\lambda<2^{\omega_{1}}$. Assume also that $2^{\aleph_{1}} \geqslant\left(2^{\aleph_{0}}\right)^{+}$, and strictly greater if $\operatorname{cf}\left(2^{\aleph_{0}}\right)=\omega_{1}$. Then there is an inner model of set theory containing a measurable cardinal.

Having shown that large cardinals are necessary to obtain some weak saturation while separating $2^{\aleph_{0}}$ from $2^{\aleph_{1}}$, we conclude this section by showing that they also suffice.

THEOREM 5.3. Suppose $\kappa \leqslant \lambda<\mu$ where $\kappa$ is supercompact, $\operatorname{cf}(\lambda)>\omega$ and $\operatorname{cf}(\mu)>\kappa$. Then there is a generic extension of the universe in which every cardinal $\geqslant \kappa$ is preserved, $2^{\aleph_{0}}=\lambda, 2^{\aleph_{1}}=\mu$ and every ideal on $\omega_{1}$ is $\omega_{3}$-saturated.

Proof. Using methods of Silver or Laver (see $\left[\mathbf{M}_{2}\right]$ and $[\mathbf{L}]$ ) it is possible to find an extension $V_{1}$ of the universe in which $\kappa$ is still supercompact, $2^{\kappa}=\mu, 2^{\aleph_{0}}=\aleph_{1}$ and $2^{\aleph_{1}}=\boldsymbol{\kappa}_{2}$. Now, using techniques of Magidor $\left[\mathbf{M}_{1}\right]$, one can find a further extension $V_{2}$ in which $\mathrm{cf}(\kappa)=\omega_{1}, \kappa$ is still a limit cardinal, and no new subsets of $\omega_{1}$ are added. Finally, we obtain the desired generic extension by adding $\lambda$ Cohen reals to $V_{2}$. Since $2^{\aleph_{1}}=\boldsymbol{\aleph}_{2}$ in $V_{2}$, Theorem 4.1 guarantees that in the extension every ideal on $\omega_{1}$ is $\omega_{3}$-saturated. The reader can now easily check that in the extension we also have that $2^{\kappa_{0}}=\lambda$ and $2^{\kappa_{1}}=2^{\kappa}$.

In Theorem 5.3 it is even possible to arrange that $\kappa=\boldsymbol{\kappa}_{\omega_{1}}$ in the extension, by using the methods of Magidor $\left[\mathbf{M}_{\mathbf{2}}\right]$. In this case, however, the known possibilities for $2^{\kappa}$ are narrowly restricted.

6. Negative preservation results for c.c.c. extensions. We showed in $\$ 2$ that if $M[G]$ is obtained from a model $M$ of $\mathrm{GCH}$ via any c.c.c. notion of forcing $P$, then in $M[G]$ every $\omega_{2}$-generated ideal on $\omega_{1}$ is $\omega_{3}$-saturated. In $\$ 4$ we showed that if the chain condition requirements on $P$ are strengthened from "c.c.c." to " $\sigma$-finite c.c.," then one can conclude that in $M[G]$ every ideal on $\omega_{1}$ is $\omega_{2}$-generated and hence every ideal on $\omega_{1}$ is $\omega_{3}$-saturated. These results suggest two natural questions. First of all, can we get the stronger saturation conclusion (i.e. the $\omega_{3}$-saturation of every ideal on $\omega_{1}$ ) from the weaker chain condition hypothesis (i.e. the c.c.c. instead of the $\sigma$-finite c.c.)? Secondly, does the converse to the first mentioned result above hold? That is, if $P$ has the c.c.c., then is it the case that in the extension the $\omega_{3}$-saturated ideals on $\omega_{1}$ are precisely the $\omega_{2}$-generated ideals on $\omega_{1}$ ?

In this seciton we answer both questions in the negative by employing a variant of a partial order constructed by Galvin and Hajnal to show that the countable chain condition is not equivalent to the $\sigma$-finite chain condition. Let $(A, E)$ be a graph (i.e. $A$ is a set and $\left.E \subseteq[A]^{2}\right)$. Let $P(A, E)=\left\{X \in[A]^{<\omega}:[X]^{2} \cap E=0\right\}$. The elements of $P(A, E)$ are usually called independent sets. Now order $P(A, E)$ by setting $x \leqslant y$ iff $y \subseteq x$. 
Theorem 6.1 (Galvin-Hajnal). Let $\kappa$ be a cardinal. Suppose $(\kappa, E)$ is a graph such that $\left(\exists \alpha<\omega_{1}\right)(\forall \xi<\kappa)\{\eta<\xi:\{\eta, \xi\} \in E\}$ has order-type at most $\alpha$. Then $P(\kappa, E)$ has the countable chain condition.

Proof. Suppose $A \subseteq P(\kappa, E)$ is an uncountable antichain. Without loss of generality we can assume that the elements of $A$ all have the same cardinality and that they form a $\Delta$-system. By subtracting off the kernel of the $\Delta$-system (the result of which is still an antichain), we can assume that $\left\langle a_{\gamma}: \gamma<\omega_{1}\right\rangle$ enumerates $A$, $\left\langle\xi_{i}^{\gamma}: i<n\right\rangle$ enumerates $a_{\gamma}$, and if $\gamma, \delta<\omega_{1}$ and $i<n$ then $\xi_{i}^{\gamma}<\xi_{i}^{\delta}$ iff $\gamma<\delta$. (For the last clause, apply the partition relation $\kappa \rightarrow(\kappa, \omega)^{2} n$ times.)

For each $\gamma<\omega_{1}$ let $X_{\gamma}=\left\{\delta<\omega_{1}:\left(\exists \xi \in a_{\delta}\right)\left(\exists \eta \in a_{\gamma}\right) \xi<\eta\right.$ and $\left.\{\xi, \eta\} \in E\right\}$. By hypothesis $X_{\gamma}$ is countable. Hence there exists an ordinal $\beta$ such that for all $\gamma<\beta$, $X_{\gamma} \subseteq \beta$ and $\beta \geqslant \alpha \cdot \omega$ (here $\alpha \cdot \omega$ denotes ordinal multiplication). Fix $\xi \in a_{\beta}$ and $i<n$. Then $B_{\xi i}=\left\{\gamma<\beta: \xi_{i}^{\beta}<\xi\right.$ and $\left.\left\{\xi_{i}^{\gamma}, \xi\right\} \in E\right\}$ must have order-type at most $\alpha$. Thus $B=\bigcup\left\{B_{\xi i}: \xi \in a_{\beta}, i<n\right\}$ must have order-type less than $\alpha \cdot \omega$, so there is $\delta<\beta$ which is not contained in $B$. But since $\beta \notin X_{\delta}$, it is clear that $a_{\delta}$ and $a_{\beta}$ are compatible, contradiction.

Now suppose that $\kappa^{\omega}=\kappa$ (although a weaker property will suffice; see the discussion concerning Martin's Axiom immediately following the proof of Theorem 6.2). For each $\alpha<\omega_{1}$ let $\mathcal{G}_{\alpha}=\left(\kappa, E_{\alpha}\right)$ be a graph such that for every $\xi<\kappa$ we have $\left\{\eta<\xi:\{\eta, \xi\} \in E_{\alpha}\right\}$ has order-type at most $\alpha$, and such that for every $X \subseteq \kappa$, if $X$ has order-type at most $\alpha$ then there are arbitrarily large $\xi$ such that $X=\{\eta<$ $\left.\xi:\{\eta, \xi\} \in E_{\alpha}\right\}$. Define $\mathcal{G}=\left(\omega_{1} \times \kappa, E\right)$ by letting $\{(\alpha, \xi),(\beta, \eta)\} \in E$ iff $\alpha=\beta$ and $\{\xi, \eta\} \in E_{\alpha}$. (Thus $\mathcal{G}$ can be represented as the disjoint union of the $\mathcal{G}_{\alpha}$ 's for $\alpha<\omega_{1}$.) An argument similar to the proof of Theorem 3.1 will show that $P=$ $P\left(\omega_{1} \times \kappa, E\right)$ has the countable chain condition.

For the rest of this section we assume that $\kappa$ and $P$ are as above. Before proceeding with the main result of this section, we offer an alternative way to view $P$ that makes what we are doing a little more clear. The key to obtaining ideals of the desired kinds is to add generically a sequence $\left\langle A_{\xi}: \xi<\kappa\right\rangle$ of subsets of $\omega_{1}$ having the property that no $A_{\xi}$ is covered by countably many other $A_{\eta}$ 's. The ideals we want will then be (respectively) the one generated by the pairwise intersections of the $A_{\xi}$ 's and the one generated by the $A_{\xi}$ 's themselves. A forcing condition will be a finite piece of information concerning which $\alpha$ 's (for $\alpha<\omega_{1}$ ) belong to which $A_{\xi}$ 's (for $\xi<\kappa)$. But in order that the $A_{\xi}$ 's have the desired property, we need to impose some additional constraint. The graph $\mathcal{G}$ can be regarded as an auxiliary construct designed for this purpose. That is, we demand that if some condition forces $\alpha$ to be in both $A_{\xi}$ and $A_{\eta}$, then $\xi$ and $\eta$ should be independent (i.e. not adjacent) in the graph. With this motivation, one is easily led to the above stated requirements of the graphs $\mathcal{G}_{\alpha}$, i.e. that every $X \subseteq \kappa$ of order type $\alpha$ is (simultaneously) adjacent to cofinally many vertices in $\mathcal{G}_{\alpha}$.

THEOREM 6.2. (a) $\mathbb{}_{P}$ "There is an ideal on $\omega_{1}$ that is not $\kappa$-saturated."

(b) If $2 \aleph_{1}=\aleph_{2}$ then $\Vdash_{P}$ "There is an ideal on $\omega_{1}$ which is $\omega_{3}$-saturated but is not generated by fewer than $\kappa$ sets." 
Proof. Suppose $G$ is $P$-generic over $V$. Then $\cup G$ is a maximal independent set in $\left(\omega_{1} \times \kappa, E\right)$. Working in $V[G]$ let $A=\left\{\xi<\omega_{1}:(\alpha, \xi) \in \cup G\right\}$. Let $\dot{A}_{\xi}$ be the canonical term denoting $A_{\xi}$.

Claim 1. $\Vdash_{P}$ " $\forall X \in[\kappa]^{\omega} \forall \xi \in \kappa-X, \dot{A}_{\xi} \nsubseteq \cup\left\{\dot{A}_{\eta}: \eta \in X\right\}$."

Proof. Since $P$ has the c.c.c. every countable $X \subseteq \kappa$ in $V[G]$ is contained in some countable set $Y$ lying in $V$. Fix such $Y$ and $\xi \in \kappa-Y$. Suppose $p \in P$. We must find $q \leqslant p$ and $\beta<\omega_{1}$ so that $q \Vdash \beta \in \dot{A}_{\xi}-\bigcup\left\{\dot{A}_{\eta}: \eta \in Y\right\}$. Let the order-type of $Y$ be $\alpha$. Choose $\beta$ so large that $p \subseteq \beta \times \kappa$ and $\alpha \leqslant \beta$. Find $\xi^{\prime}$ so that $Y=\left\{\eta<\xi^{\prime}:\left\{\eta, \xi^{\prime}\right\}\right.$ $\left.\in E_{\beta}\right\}$. Now let $q=p \cup\left\{(\beta, \xi),\left(\beta, \xi^{\prime}\right)\right\}$. Then $q$ works, and the claim is established.

Let $\dot{I}$ denote the ideal generated by the sets $\dot{A}_{\xi} \cap \dot{A}_{\eta}, \xi \neq \eta$. By Claim 1, $\mathbb{}_{P} \forall \xi<\kappa\left(\dot{A}_{\xi} \in \dot{I}^{+}\right)$. Thus $\mathbb{\Vdash}_{P}$ " $\dot{I}$ is not $\kappa$-saturated," and (a) is proved.

For (b), we let $\dot{I}$ denote the ideal generated by the sets $A_{\xi}$ for $\xi<\kappa$. By Claim 1, $\mathbb{}_{P}$ " $I$ is not generated by fewer than $\kappa$ sets."

Suppose $p \vDash\left\langle\dot{B}_{\gamma}: \gamma<\omega_{3}\right\rangle$ is an $\dot{I}$-almost disjoint family of elements of $\dot{I}^{+}$." We seek a contradiction. For simplicity in what follows, we assume $p=\varnothing$.

Let us call a set $X \subseteq \omega_{1} \times \kappa$ closed if for every $(\alpha, \xi) \in X$ and for every $\eta<\xi$, if $\{(\alpha, \xi),(\alpha, \eta)\} \in E$ then $(\alpha, \eta) \in X$. By construction of $E$ every countable set is contained in a countable closed set.

Claim 2. Fix $\gamma<\omega_{3}$. There is a set $I_{\gamma} \subseteq \kappa$ such that $\left|I_{\gamma}\right|=\omega_{1}, \omega_{1} \times I_{\gamma}$ is closed, and for every countable $X \subseteq I_{\gamma}$ there is $p \subseteq \omega_{1} \times I_{\gamma}$ and arbitrarily large $\beta$ such that $p \Vdash \beta \in \dot{B}_{\gamma}-\bigcup\left\{\dot{A}_{\xi}: \xi \in X\right\}$.

Proof. We will obtain $I_{\gamma}$ as the union of an increasing continuous sequence $\left\langle X_{\alpha}: \alpha<\omega_{1}\right\rangle$. Given $X_{\alpha}$, find $p$ and $\beta>\alpha$ so that $p \Vdash \beta \in \dot{B}_{\gamma}-\cup\left\{\dot{A}_{\xi}: \xi \in X_{\alpha}\right\}$. Choose $X_{\alpha+1}$ so that $p \subseteq \omega_{1} \times X_{\alpha+1}$ and for some closed set $Y$ we have $\alpha \times X_{\alpha} \subseteq Y$ $\subseteq \omega_{1} \times X_{\alpha+1}$.

By Claim 2 and the assumption that $2^{\aleph_{1}}=\boldsymbol{\aleph}_{2}$ there is $Z \subseteq \omega_{3}$ such that $|Z|=\boldsymbol{\aleph}_{3}$ and $\left\{I_{\gamma}: \gamma \in Z\right\}$ forms a $\Delta$-system with kernel $K$. Let us call $\gamma, \delta \in Z$ isomorphic if there is a bijection $f: I_{\gamma} \rightarrow I_{\delta}$ such that $f \mid K$ is the identity and the natural lifting of $f$ to $\omega_{1} \times I_{\gamma}$ and $\left[\omega_{1} \times I_{\gamma}\right]^{<\omega}$ satisfies the following.

(1) $\forall p \in\left[\omega_{1} \times I_{\gamma}\right]^{<\omega}(p \in P$ iff $f(p) \in P)$.

(2) $\forall p \in\left[\omega_{1} \times I_{\gamma}\right]^{<\omega} \forall \beta<\omega_{1}\left(p \Vdash \beta \in \dot{B}_{\gamma}\right.$ iff $\left.f(p) \Vdash \beta \in \dot{B}_{\delta}\right)$.

It is easy to see that the total number of isomorphism types is at most $2 \boldsymbol{N}_{1}=\boldsymbol{\aleph}_{2}$, so there must be isomorphic $\gamma, \delta \in Z$ with $\gamma \neq \delta$. Let $f: I_{\gamma} \rightarrow I_{\delta}$ be the isomorphism.

Now $\Vdash_{P} \dot{B}_{\gamma} \cap \dot{B}_{\delta} \in \dot{I}$, so since $P$ has the c.c.c. there is a countable set $X \in V$ such that $\Vdash_{P} \dot{B}_{\gamma} \cap \dot{B}_{\delta} \subseteq \cup\left\{\dot{A}_{\xi}: \xi \in X\right\}$. Let $\bar{X}=\left(X \cap I_{\gamma}\right) \cup f^{-1}\left(X \cap I_{\delta}\right)$. By constuction of $I_{\gamma}$ there is $p \subseteq \omega_{1} \times I_{\gamma}$ and $\beta$ such that $\beta$ is greater than or equal to the order-type of $\overline{\bar{X}}$ and $p \Vdash \beta \in \dot{B}_{\gamma}-\cup\left\{\dot{A}_{\xi}: \xi \in \bar{X}\right\}$. Note in particular that $p \cap(\{\beta\} \times \bar{X})=0$. Therefore $f(p) \cap(\{\beta\} \times f(\bar{X}))=0$ so $(p \cup f(p)) \cap$ $(\{\beta\} \times \bar{X})=0$. By (2), $f(p) \Vdash \beta \in \dot{B}_{\delta}$. If $p$ and $f(p)$ are compatible, then letting $\xi$ be such that $\{\eta<\xi:\{(\beta, \eta),(\beta, \xi)\} \in E\}=X$, we have

$$
q=p \cup f(p) \cup\{(\beta, \xi)\} \in P \text { and } q \Vdash \beta \in\left(\dot{B}_{\gamma} \cap \dot{B}_{\delta}\right)-\cup\left\{\dot{A}_{\xi}: \xi \in X\right\} \text {, }
$$

a contradiction. 
So suppose $(\alpha, \xi) \in p,(\alpha, \eta) \in f(p)$ and $\{(\alpha, \xi),(\alpha, \eta)\} \in E$. If $\xi<\eta$ then since $I_{\delta}$ is closed we have $(\alpha, \xi) \in \omega_{1} \times I_{\delta}$. But then $(\alpha, \xi) \in \omega_{1} \times K$ and $f$ is the identity on $(\alpha, \xi)$ so $(\alpha, \xi) \in f(p)$, a contradiction. A similar contradiction is reached if $\eta<\xi$. This completes the proof of Theorem 6.2.

Theorem 4.2 showed that it is relatively consistent with $M A_{\omega_{2}}$ that all ideals on $\omega_{1}$ are $\omega_{3}$-saturated. Theorem 6.2 yields a further clarification of the role played by Martin's Axiom as shown by the following.

COROLLARY 6.3. (a) It is relatively consistent with $M A_{\kappa}$ that non- $\kappa$-saturated ideals on $\omega_{1}$ exist.

(b) The existence of non- $\omega_{3}$-saturated ideals on $\omega_{1}$ is independent of $M A_{\omega_{2}}$.

(c) If $\omega_{3} \leqslant \kappa<\omega_{\omega}$ then $M A_{\kappa}$ implies there is a non- $\kappa$-saturated ideal on $\omega_{1}$.

Proof. For part (a) we force first with the $P$ of this section and then force $M A_{\kappa}$ in the usual c.c.c. fashion. Part (b) follows immediately from part (a) with $\kappa=\omega_{2}$, and Theorem 4.2. For part (c) recall that, as remarked following Theorem 3.1, there is a stationary (hence unbounded) subset $S$ of $[\kappa]^{\omega}$ of cardinality $\kappa$. Now if we construct the graphs $\mathcal{G}_{\alpha}=\left(\kappa, E_{\alpha}\right)$ with the property that for any $X \in S$, if $X$ has order-type at most $\alpha$, then for any finite set $F \subseteq \kappa$ there are arbitrarily large $\xi<\kappa$ such that $\left\{\eta<\xi:\{\eta, \xi\} \in E_{\alpha}\right\}=X-F$, we see that the resulting version of $P\left(\omega_{1} \times \kappa, E\right)$ still satisfies Theorem 3.2, and it has cardinality $\kappa$ so $M A_{\kappa}$ applies to it.

Theorem 6.2 shows that $P$ forces the existence of a non- $\kappa$-saturated ideal on $\omega_{1}$. The question of whether or not $P$ forces the existence of such an ideal that is normal turns out to be more complicated, and its consideration requires a few preliminaries.

If $f, g \in{ }^{\omega_{1}} \omega_{1}$, then $g$ eventually dominates $f$, written $f \ll g$, iff $\left\{\alpha \in \omega_{1}: f(\alpha)<\right.$ $g(\alpha)\}$ contains a closed unbounded set. There is a canonical minimal sequence $\left\langle f_{\alpha}: \alpha<\omega_{2}\right\rangle$ of functions increasing under eventual dominance, which may be obtained as follows. For $\alpha<\omega_{2}$ let $k_{\alpha}: \alpha \rightarrow \omega_{1}$ be one-to-one. Then let $f_{\alpha}(\beta)$ be the order-type of $k_{\alpha}^{-1}(\beta)$ for each $\beta<\omega_{1}$. It is well known and easy to check that if $\alpha<\beta$ then $f_{\alpha} \ll f_{\beta}$. Moreover, if $\left\langle g_{\alpha}: \alpha<\omega_{2}\right\rangle$ is an increasing sequence under eventual dominance, then for every $\alpha<\omega_{2}$ we have $\left\{\beta<\omega_{1}: f_{\alpha}(\beta) \leqslant g_{\alpha}(\beta)\right\}$ contains a closed unbounded set, but we will not need this fact.

The sequence $\left\langle g_{\alpha}: \alpha<\omega_{2}\right\rangle$ is called a scale if for every $f: \omega_{1} \rightarrow \omega_{1}$ there is some $\alpha<\omega_{2}$ such that $f \ll g_{\alpha}$.

THEOREM 6.4. (a) If the sequence $\left\langle f_{\alpha}: \alpha<\omega_{2}\right\rangle$ defined above is not a scale, then $\Vdash_{P}$ "There is a normal ideal on $\omega_{1}$ which is not $\kappa$-saturated."

(b) Assume $2^{\aleph_{1}}=\boldsymbol{\aleph}_{2}$. If $\left\langle f_{\alpha}: \alpha<\omega_{2}\right\rangle$ is a scale, then $\mathbb{}_{P}$ " Every normal ideal on $\omega_{1}$ is $\omega_{2}$-generated and $\omega_{3}$-saturated."

Proof. (a) Fix $f: \omega_{1} \rightarrow \omega_{1}$ so that for all $\alpha<\omega_{2}$, it is not true that $f \ll f_{\alpha}$. If $G$ is $P$-generic over $V$, then in $V[G]$ we define sets $A_{\xi}$ for $\xi<\kappa$ by $A_{\xi}=\{\alpha<$ $\left.\omega_{1}:(f(\alpha), \xi) \in \cup G\right\}$. Let $\dot{A}_{\xi}$ be the canonical term denoting $A_{\xi}$, and let $I$ denote the smallest normal ideal containing the sets $\dot{A}_{\xi} \cap \dot{A}_{\eta}$ for $\xi \neq \eta$. To show that $\Vdash_{P}$ " $\dot{I}$ is not $\kappa$-saturated," it will suffice to prove the following claim. 
Claim. $\mathbb{}_{P}\left(\forall X \in[\kappa]^{\omega_{1}}\right)(\forall \xi \in \kappa-X)\left(\dot{A}_{\xi} \nsubseteq \nabla\left\{\dot{A}_{\eta}: \eta \in X\right\}\right)$.

Proof. Since $P$ has the c.c.c. every $X \in[\kappa]^{\omega_{1}} \cap V[G]$ must be contained in some $Y \in[\kappa]^{\omega_{1}} \cap V$. Fix such $Y \in[\kappa]^{\omega_{1}} \cap V$ and let $\left\langle\xi_{\beta}: \beta<\alpha\right\rangle$ enumerate $Y$ in increasing order. Let $\dot{Z}$ be a term denoting $\left\{\gamma<\omega_{1}:(\forall \xi<\gamma)\left(\gamma \in \dot{A}_{\xi_{\zeta}}\right.\right.$ and $\left.\left.k_{\alpha}(\zeta)=\delta\right)\right\}$. Then $\dot{Z}$ denotes one possible diagonal union of $\left\{\dot{A}_{\xi}: \xi \in Y\right\}$. Other diagonal unions may differ from this one by a nonstationary set, but every nonstationary set in $V[G]$ is contained in a nonstationary set from $V$. Thus, if $N \in V$ is nonstationary, it will suffice to prove that for $\xi \in \kappa-Y$ we have $\Vdash_{P} \dot{A}_{\xi} \subseteq \dot{Z} \cup N$.

Let $p \in P$. Since it is not true that $f \ll f_{\alpha}$, there is some $\beta \notin N$ such that $f(\beta) \geqslant f_{\alpha}(\beta)$ and $p \subseteq \beta \times \kappa$. (We assume $f(\beta) \geqslant \beta$ for all $\beta$.) Now $\left\{\xi_{\xi}: k_{\alpha}(\zeta)<\beta\right\}$ has order-type $f_{\alpha}(\beta) \leqslant f(\beta)$; hence there is $\xi^{\prime}$ such that

$$
\left\{\eta<\xi^{\prime}:\left\{(f(\beta), \eta),\left(f(\beta), \xi^{\prime}\right)\right\} \in E\right\}=\left\{\xi_{\zeta}: k_{\alpha}(\zeta)<\beta\right\} .
$$

Let $q=p \cup\left\{(f(\beta), \xi),\left(f(\beta), \xi^{\prime}\right)\right\}$. Then clearly $q \Vdash \beta \in \dot{A}_{\xi}-(\dot{Z} \cup N)$. This proves (a).

The proof of (b) is quite similar to the proof of Theorem 4.1 (with $\kappa=\omega_{1}$ ), so we only outline it. Let $\left\langle\dot{A}_{\alpha}: \alpha<\lambda\right\rangle, \mathscr{Q}$ and $\mathscr{B}$ be as in the latter proof, except that this time we must choose $\mathscr{B}$ to be relatively $\omega_{2}$-saturated, and $\mathscr{Q}$ must be enlarged a little so that several additional facts mentioned below are expressible in it.

We assert that for every $\alpha<\lambda$ there is $f: \omega_{1} \rightarrow \mathscr{B} \cap \lambda$ and nonstationary $N$ such that $f, N \in V$ and

$$
\text { It } \dot{A}_{\alpha} \subseteq\left\{\xi:(\exists \eta<\xi)\left(\xi \in \dot{A}_{f(\eta)}\right)\right\} \cup N .
$$

Since $2^{\aleph_{1}}=\aleph_{2}$ in $V$, this will prove that $I$ is $\omega_{2}$-generated.

Now fix $\alpha \in \lambda$. If $\alpha \in \mathscr{B} \cap \lambda$ we are done, so assume not. Let $\left\langle\alpha_{\gamma}: \gamma<\omega_{2}\right\rangle$ be such that $\alpha_{\gamma}$ realizes the same type over $\left\langle\mathscr{B}, \alpha_{\delta}\right\rangle_{\delta<\gamma}$ that $\alpha$ realizes. For each $\delta<\omega_{1}$ let $M_{\xi}^{\alpha}$ be a maximal incompatible subset of $\left\{p \in P: p \Vdash \xi \in \dot{A}_{\alpha}\right\}$. We assume that $\mathcal{Q}$ is such that every element of $M_{\xi}^{\alpha}$ is definable in $\mathcal{Q}$ from $\alpha$ and $\xi$.

Define $g: \omega_{1} \rightarrow \omega_{1}$ so that for every $\xi$ and every $p \in M_{\xi}^{\alpha}$ we have $p \subseteq g(\xi) \times \kappa$. Since $\left\langle f_{\gamma}: \gamma<\omega_{2}\right\rangle$ is a scale, there is $\gamma$ such that $N=\left\{\xi: g(\xi) \cdot \omega>f_{\gamma}(\xi)\right\}$ is nonstationary. Here again " $g(\xi) \cdot \omega$ " denotes ordinal multiplication. Define $f: \omega_{1} \rightarrow$ $\mathscr{B} \cap \lambda$ so that $f(\xi)=\alpha_{\delta}$, where $k_{\gamma}(\delta)=\xi$. We claim that (1) holds.

Suppose $\xi \notin N$ and $q \Vdash \xi \in \dot{A}_{\alpha}$. We must show that $q \Vdash(\exists \eta<\xi)\left(\xi \in \dot{A}_{f(\eta)}\right)$. There is $p \in M_{\xi}^{\alpha}$ compatible with $q$, and since $p$ is definable in $\mathcal{Q}$ from $\alpha$ and $\xi$ there is a sequence $\left\langle p_{\delta}: \delta\langle\gamma\rangle\right.$ such that each $p_{\delta}$ has the same properties with respect to $\left\langle p_{\delta^{\prime}}: \delta^{\prime}<\delta\right\rangle$ that $p$ has. In particular, we must have $p=\left\langle\left(\xi_{1}, \beta_{1}^{\delta}\right), \ldots,\left(\xi_{n}, \beta_{n}^{\delta}\right)\right\rangle$ where $n$ and the $\xi_{i}$ are the same for all $\delta$. Also, $p=\left\langle\left(\xi_{1}, \beta_{1}\right), \ldots,\left(\xi_{n}, \beta_{n}\right)\right\rangle$ and for each $i\left\langle\beta_{i}^{\delta}: \beta_{i}^{\delta}<\beta_{i}\right\rangle$ is a strictly increasing sequence while $\left\langle\beta_{i}^{\delta}: \beta_{i}^{\delta}>\beta_{i}\right\rangle$ is strictly decreasing, hence finite.

If $\left(\xi^{\prime}, \beta\right) \in p \cup q$ then $B_{i}=\left\{\beta_{i}^{\delta}<\beta:\left\{\left(\xi_{i}, \beta_{i}^{\delta}\right),\left(\xi^{\prime}, \beta\right)\right\} \in E\right\}$ has order-type at most $\xi^{\prime}$; hence by the remarks above $B=\left\{\xi: \exists i\left(\beta_{i}^{\delta} \in B_{i}\right)\right\}$ has order-type $<\xi^{\prime} \cdot \omega$ $\leqslant g(\xi) \cdot \omega \leqslant f_{\gamma}(\xi)$, and by definition of $f$ and $f_{\gamma},\left\{\delta: k_{\gamma}(\delta)<\xi\right\}$ has order-type $f_{\gamma}(\xi)$ and so there is $\delta$ such that $k_{\gamma}(\delta)<\xi$ and $\delta \notin B$. But now it is easy to see that $p_{\delta} \cup p \cup q$ is compatible, and this completes the proof. 
By combining the proofs of Theorem 6.2(b) and Theorem 6.4(a) we arrive at the following theorem. Details are left to the reader.

THEOREM 6.5. If $2^{\aleph_{1}}=\aleph_{2}$ and $\left\langle f_{\alpha}: \alpha<\omega_{2}\right\rangle$ is a scale, then $\mathbb{t}_{P}$ "There is a normal ideal on $\omega_{1}$ which is $\omega_{3}$-saturated but is not generated by fewer than $\kappa$ sets."

REMARK. It is well known that if $N S_{\omega_{1}}$ is $\omega_{2}$-saturated, then $\left\langle f_{\alpha}: \alpha<\omega_{2}\right\rangle$ is a scale. The converse seems to be open, although it is well known, for example, that $\diamond$ implies that $\left\langle f_{\alpha}: \alpha<\omega_{2}\right\rangle$ is not a scale. It will be shown in [BT] that if $N S_{\omega_{1}}$ is $\omega_{2}$-saturated then $\Vdash_{P}$ " $N S_{\omega_{1}}$ is $\omega_{2}$-saturated," and it follows from [BTW $\left.\mathbf{W}_{1}\right]$ that $\Vdash_{P}$ "Every normal ideal on $\omega_{1}$ is $\omega_{2}$-saturated."

7. Questions. In this section we will discuss several questions suggested by the results in $\$ \S 1$ through 6 . For the sake of clarity we will generally state the simplest case of the questions we have been unable to settle. As a beginning, let us record an instance of the remark following Theorem 2.1.

Question 7.1. Suppose that every ideal on $\omega_{1}$ is $\omega_{3}$-saturated. Does it follow that every ideal on $\omega_{1}$ is $\omega_{2}$-generated?

It is easy to see that many of our results generalize in a natural way to the context of $\mu$-chain condition partial orderings. For example, this is true of Theorems 2.2-2.4, Theorem 3.1 and Theorem 4.1. On the other hand, we are unable to generalize Theorems $3.2-3.5$ to the nonstationary ideal on $[\kappa]^{\omega_{1}}$. In fact, it turns out that the obvious generalization of Corollary 3.3 to cardinals greater than $\omega$ fails. One can show much as in Theorem 3.2(a) that if $C$ is a closed unbounded subset of $\left[\omega_{3}\right]^{\omega_{1}}$, then there is a set $A \in\left[\omega_{2}\right]^{\omega_{1}}$ such that $\left|[A]^{\omega_{1}} \cap C\right| \geqslant 2^{\kappa_{0}}$. In a preliminary version of this paper we asked whether it was provable in ZFC that every closed unbounded subset of $\left[\omega_{3}\right]^{\omega_{1}}$ has cardinality $\max \left(\boldsymbol{\kappa}_{3}, 2^{\boldsymbol{\kappa}_{1}}\right)$. The referee kindly supplied us with a model for the negative answer in which $2^{\boldsymbol{\aleph}_{0}}=\boldsymbol{\aleph}_{\omega_{1}}$ and $2^{\boldsymbol{\aleph}_{1}}=\boldsymbol{\aleph}_{\omega_{1}+1}$. Moreover, recent results of Avraham and the first named author yield the consistency of a general negative answer. It also turns out that a positive solution is consistent (assuming the consistency of an Erdös cardinal). These results will appear elsewhere.

Our considerations of saturated ideals and the $\mathrm{GCH}(\S 5)$ bring to mind the following question.

Question 7.2. Suppose $2^{\aleph_{0}}=\boldsymbol{\aleph}_{1}$ and $2^{\boldsymbol{\aleph}_{1}}=\boldsymbol{\aleph}_{3}$. Can one then prove that $N S_{\omega_{1}}$ is not $\omega_{3}$-saturated?

In Corollary 6.3 we showed that it is relatively consistent with $M A_{\kappa}$ that non- $\kappa$ saturated ideals on $\omega_{1}$ exist, but we were only able to prove that $M A_{\kappa}$ directly implies this for $\omega_{3} \leqslant \kappa<\omega_{\omega}$. This suggests two questions (where an affirmative answer to the first would yield an affirmative answer to the second).

Question 7.3. Is there a stationary (or unbounded) subset of $\left[\boldsymbol{\aleph}_{\omega+1}\right]^{\omega}$ of cardinality $\boldsymbol{N}_{\omega+1}$ ?

Question 7.4. Does $M A_{\kappa}$ for $\kappa=\boldsymbol{\aleph}_{\omega+1}$ imply that there is a non- $\kappa$-saturated ideal on $\omega_{1}$ ?

Difficulties also arise in trying to extend Theorems 6.1 and 6.2 to $\omega_{2}$-complete ideals on $\omega_{2}$. If one tries to generalize $P(A, E)$ using finite independent sets, then $\omega_{1}$ is collapsed, while if countable independent sets are used the $\omega_{2}$-chain condition fails 
because of the Milner-Rado "paradox" [MR], which asserts that every $\alpha<\omega_{2}$ is the union of countably many sets, each of order-type less than $\omega_{1}^{\omega}$. Hence, we are left with the following.

Question 7.5. Does there exist an $\omega_{2}$-chain condition partial ordering $P$ such that $\mathbb{}_{P}$ "There is an $\omega_{2}$-complete ideal on $\omega_{2}$ that is not $\omega_{4}$-saturated?"

If $\dot{I}$ and $\left\{\dot{A}_{\xi}: \xi<\kappa\right\}$ are as in Theorem 6.2(a), then the methods of Theorem 6.2(b) can be used to show that $\mathbb{F}_{P}$ " $\dot{I} \mid \dot{A}_{\xi}$ is $\omega_{3}$-saturated." It follows that if $\omega_{3} \leqslant \lambda \leqslant \kappa$ and $\dot{I}_{\lambda}$ denotes the ideal generated by $\dot{I} \cup\left\{\dot{A}_{\xi}: \lambda \leqslant \xi<\kappa\right\}$, then $\mathbb{}_{P}$ " $\dot{I}_{\lambda}$ is $\lambda^{+}$saturated but not $\lambda$-saturated." By forcing first with $P$ (with $\kappa=\omega_{6}$, say), and then adding $\boldsymbol{\aleph}_{15}$ Cohen reals we arrive at a model in which $2^{\boldsymbol{\aleph}_{0}}=\boldsymbol{\aleph}_{15}$ and there are $\lambda^{+}$-saturated non- $\lambda$-saturated ideals on $\omega_{1}$ iff $\omega_{2} \leqslant \lambda \leqslant \omega_{6}$. Also, by adding first (via countably closed forcing) a Kurepa tree with $\aleph_{4}$ branches and then proceeding as above, we can find a model in which $2^{\boldsymbol{\aleph}_{0}}=\boldsymbol{\aleph}_{15}$ and there are $\lambda^{+}$-saturated non- $\lambda$-saturated ideals on $\omega_{1}$ iff $\omega_{4} \leqslant \lambda \leqslant \omega_{6}$. This requires noticing first (as in [JP]) that if such a Kurepa tree exists then no ideal on $\omega_{1}$ can be $\omega_{4}$-saturated; and secondly, in Theorem $2.2 \mathrm{GCH}$ was used only for the partition relation in the proof, and an appropriate version holds here without GCH. Details are omitted. We do not know whether the set of $\lambda$ 's as above must always be an interval. For example,

Question 7.6. Is it consistent that there are $\lambda^{+}$-saturated non- $\lambda$-saturated ideals on $\omega_{1}$ iff $\lambda=\omega_{2}$ or $\lambda=\omega_{4}$ ?

\section{REFERENCES}

[B $\left.\mathbf{B}_{1}\right]$ J. Baumgartner, Almost disjoint sets, the dense set problem and the partition calculus, Ann. Math. Logic 10 (1976), 401-439.

$\left[\mathbf{B}_{2}\right] \ldots$ Canonical partition relations, J. Symbolic Logic 40 (1975), 541-554.

[BT] J. Baumgartner and A. Taylor, Saturation properties of ideals in generic extensions. II, Trans. Amer. Math. Soc. (to appear).

[BTW $\mathbf{1}_{1}$ J. Baumgartner, A. Taylor and S. Wagon, On splitting stationary subsets of large cardinals, J. Symbolic Logic 42 (1977), 203-214.

$\left[\mathbf{B T W}_{2}\right] \ldots$ Structural properties of ideals, Dissertationes Math. (to appear).

[Di] C. DiPrisco, Combinatorial properties and supercompact cardinals, Ph.D. Thesis, M.I.T., 1976.

[DJ] A. Dodd and R. Jensen, The core model, circulated notes (1976).

[EHMR] P. Erdös, A. Hajnal, A. Máté and R. Rado, Combinatorial set theory: Partition relations for cardinals (to appear).

[F] G. Fodor, Eine Bemerkung zur Theorie der regressiven Funktionen, Acta Sci. Math. (Szeged) 17 (1956), 139-142.

[J] T. Jech, Some combinatorial problems concerning uncountable cardinals, Ann. Math. Logic 5 (1973), 165-198.

$\left[\mathbf{J}_{\mathbf{2}}\right]$, On the number of generators of an ideal (to appear).

[JP] T. Jech and K. Prikry, Ideals over uncountable sets: application of almost disjoint functions and generic ultrapowers, Mem. Amer. Math. Soc. no. 214 (1979).

[Je] R. Jensen, Marginalia to a theorem of Silver, circulated notes (1975).

[K] Y. Kakuda, Saturated ideals in Boolean extensions, Nagoya Math. J. 48 (1972), 159-168.

[Ka] A. Kanamori, Perfect set forcing for uncountable cardinals, Ann. Math. Logic 19 (1980), 97-114.

[Ke] J. Ketonen, Some combinatorial principles, Trans. Amer. Math. Soc. 188 (1974), 387-394.

[Ku] D. Kueker, Countable approximations and Löwenheim-Skolem theorems, Ann. Math. Logic 11 (1977), 57-103.

[Kun] K. Kunen, Saturated ideals, J. Symbolic Logic 43 (1978), 65-76.

[KP] K. Kunen and J. Paris, Boolean extensions and measurable cardinals, Ann. Math. Logic 2 (1971), 359-378. 
[L] R. Laver, Making the supercompactness of $\kappa$ indestructible under $\kappa$ directed closed forcing, Israel J. Math. 29 (1978), 385-388.

$\left[\mathbf{M}_{1}\right]$ M. Magidor, Changing cofinality of cardinals, Fund. Math. 159 (1978), 61-71.

$\left[\mathbf{M}_{2}\right] \ldots$, On the singular cardinals problem. II, Ann. of Math. (to appear).

$\left[\mathrm{Me}_{1}\right]$ T. Menas, On strong compactness and supercompactness, Ann. Math. Logic 7 (1975), 327-359.

$\left[\mathbf{M e}_{2}\right] \ldots$, Consistency results concerning supercompactness, Trans. Amer. Math. Soc. 223 (1976), $61-91$.

[MR] E. Milnor and R. Rado, The pigeon-hole principle for ordinal numbers, Proc. London Math. Soc. 15 (1965), 750-768.

[P] K. Prikry, Changing measurable into accessible cardinals, Dissertationes Math. 68 (1970), 5-52.

[S] R. Solovay, Real valued measurable cardinals, Axiomatic Set Theory, Proc. Sympos. Pure Math., vol. 13, Amer. Math. Soc., Providence, R.I., 1971, pp. 397-428.

$\left[T_{1}\right]$ A. Taylor, Regularity properties of ideals and ultrafilters, Ann. Math. Logic 16 (1979), 33-55.

$\left[\mathbf{T}_{2}\right] \ldots$, On saturated sets of ideals and Ulam's problem, Fund. Math. 109 (1980), 37-53.

[W] S. Wagon, The saturation of a product of ideals, Canad. J. Math. 32 (1980), 70-75.

Department of Mathematics, Dartmouth College, Hanover, New Hampshire 03755

Department of Mathematics, Union College, Schenectady, New York 12308 